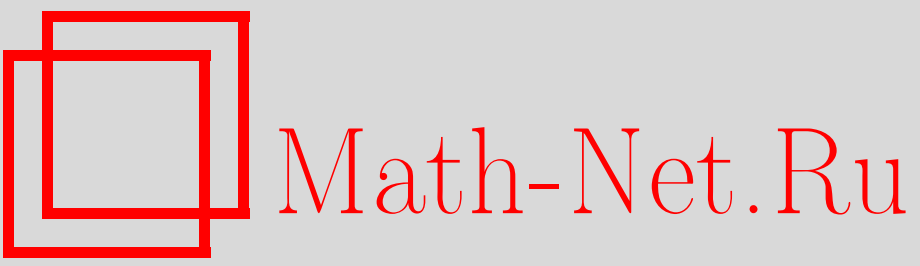

С. А. Вакуленко, П. В. Гордон, Распространение и рассеяние кинков в неоднородной нелинейной среде, ТМФ, 1997, том 112, номер 3, 384-394

DOI: https://doi.org/10.4213/tmf1050

Использование Общероссийского математического портала Math-Net.Ru подразумевает, что вы прочитали и согласны с пользовательским соглашением

http://www . mathnet.ru/rus/agreement

Параметры загрузки:

IP : 54.166 .219 .16

26 апреля 2023 г., 14:10:45 
ТЕОРЕТИЧЕСКАЯ

И МАТЕМАТИЧЕСКАЯ

ФИЗИКА

Том 112, № 3

сентябрь, 1997

\author{
С. А. Вакуленко*, П.В. Гордон*
}

\title{
РАСПРОСТРАНЕНИЕ И РАССЕЯНИЕ КИНКОВ В НЕОДНОРОДНОЙ НЕЛИНЕЙНОЙ СРЕДЕ
}

Рассмотрена модель рассеяния кинков в неоднородной нелинейной среде. Показано сушествование периодически и хаотически осциллирующих по времени связанных состояний кинков.

\section{1. ВВЕ ДЕНИЕ}

Распространение нелинейных волн - один из основных эффектов в диссипативных средах. Он наблюдается как в физике, так и в химии, биологии и экологии.

Впервые такие волны были открыты в работе [1], и с тех пор их изучение стало одной из центральных проблем, которой посвящено множество исследований, например [2-6]. Когда среда описывается скалярным параметром порядка и, следовательно, одним нелинейным параболическим уравнением, почти все уже известно. В этом случае волны в однородной одномерной среде имеют вид кинков $u(x-v t)$, где $v$ - скорость $[7,8]$.

Теория распространения и рассеяния таких волн в неоднородных средах также хорошо развита. Для описания движения их фронтов был разработан асимптотический подход $[2,4]$.

Значительно сложнее обстоит дело, когда параметр порядка - вектор, т.е. мы имеем дело с системой нелинейных уравнений параболического типа. Известно, что при некоторых ограничениях на вид нелинейности (случай так называемых монотонных систем) кинки имеют вид бегущих волн [9]. Можно показать, что в общем случае это не так: волны вида $u(x-v t)$ могут не существовать $[4,10,11]$. Кроме того, для немонотонных систем может иметь место ряд новых физических эффектов, отсутствуюших в случае одного уравнения или монотонной системы $[4,10,11]$. Некоторые из них были предсказаны в $[4,10]$, однако там не было найдено систем явного и простого вида, которые описывают эти явления.

Цель настояшей работы - построить такие модели и описать новые эффекты, в частности:

I. Сушествование даже в однородной среде связанных состояний кинков с периодически и хаотически осциллируюшей структурой, которые могут двигаться как целое с переменной скоростью.

* Институт проблем машиноведения РАН, Санкт-Петербург, Россия. E-mail:

vakul@microm.ipme.ru; gordon@mech.ipme.ru 
II. Смену типа осцилляций в таких состояниях при прохождении волной неоднородности среды.

III. Возможный распад связанного состояния на отдельные волны (кинки) при прохождении волной неоднородности среды или, наоборот, образование связанного состояния из первоначально независимых фронтов.

Отметим, что некоторые эффекты взаимодействия кинков здесь напоминают аналогичные эффекты, возникающие в уравнении синус-Гордон, где возможно образование бризера - периодически осциллируюшего связанного состояния двух солитонов. В нашем случае, однако, возможны и хаотические осцилляции.

Исследуемая модель имеет вид

$$
\frac{\partial u_{i}}{\partial t}=d_{i} \frac{\partial^{2} u_{i}}{\partial x^{2}}+g\left(u_{i}\right)+\epsilon f_{i}(\mathbf{u}, x)
$$

где $x \in \mathbf{R}$, параметр порядка $\mathbf{u}=\left(u_{1}, u_{2}, \ldots, u_{n}\right)$ состоит из $n$ компонент и $\epsilon$ - малый параметр, $f_{i}, g \in C^{3}$.

В простейшем случае

$$
d_{i}=1, \quad g(u)=2\left(u-u^{3}\right),
$$

однако вся схема анализа может быть применена для (1.1) с нелинейностями $g$ более обшего вида и для некоторых других уравнений, которые могут возникать в гидродинамике, теории плазмы и других областях. Например, слагаемое $g\left(u_{i}\right)$ может быть заменено выражением вида $u_{i} \partial u_{i} / \partial x ; f_{i}$ могут содержать первые производные от $\mathbf{u}$ и т.д.

Тем не менее ради простоты формул предполагаем всюду ниже, что вьполнено (1.2).

В отличие от $[10,11]$ возмушения $f_{i}$, описываюшие взаимодействие между компонентами $u_{i}$, имеют простую форму: это линейные или квадратичные функции $u_{i}$ с коэффициентами, зависящими от $x$.

Кроме того, мы кратко опишем сравнительно новый подход к изучению движения и рассеяния таких волн, который можно было бы назвать нелинейным аналогом метода Ритца-Галеркина, и результаты численных экспериментов.

Статья построена следуюшим образом. В разделе 2 мы излагаем результаты работ $[10,11]$, в разделе 3 исследуем простейшую модель, описываюшую периодический эффект І в случае однородной среды. В разделе 4 приводим систему, описывающую эффекты I, II для случая неоднородной среды. В разделе 5 даем пример образования хаотических связанных состояний кинков. Далее в разделе 6 мы приводим пример системы, описьвающей эффект III. Последняя часть работы содержит результаты численных экспериментов, которые подтверждают аналитические выводы и показывают, что происходит при немалых $\epsilon$.

\section{2. МЕТОД ИССЛЕДОВАНИЯ СИСТЕМЫ (1.1) И ЕЕ ОБШИЕ СВОЙСТВА}

В этом разделе мы кратко изложим метод и результаты, детально представленные в $[10,11]$. Этот метод позволяет описать поведение исходной системы дифференциальных уравнений в частных производных (УЧП) с помощью значительно более простой системы обыкновенных дифференциальных уравнений (ОДУ) первого порядка. Получаемая система ОДУ описывает эволюцию координат кинков.

2 Теоретическая и математическая физика, т. 112, № 3, 1997 г. 
Будем искать решение системы (1.1) в следуюшем виде:

$$
u_{i}(x, t)=U_{i}\left(x-q_{i}(t)\right)+v_{i}(x, t), \quad \mathbf{U}(x, \mathbf{q})=\left(U_{1}, \ldots, U_{n}\right), \quad \mathbf{q}=\left(q_{1}, \ldots, q_{n}\right),
$$

где вектор-функция $\mathbf{U}$ - волновое решение невозмушенной системы (1.1) $(\epsilon=0)$. В частности, для рассматриваемого в данной работе типа нелинейности (1.2) имеем

$$
U_{i}(x)=\operatorname{th}\left(x-q_{i}\right), \quad q_{i}=\text { const }, \quad i=1,2, \ldots, n .
$$

Пусть, далее, начальные данные для уравнения (1.1) имеют форму (2.1), где функции $v_{i}(x, t)$ в начальный момент времени $(t=0)$ удовлетворяют оценке

$$
\left\|v_{i}(\cdot, t)\right\|<C \epsilon^{\kappa}, \quad \kappa>0
$$

|| || есть $L_{2}$-норма в $\mathbf{R}, C$ - некоторая константа. С помошью условия (2.3) нетрудно доказать глобальную разрешимость, $C^{2,1}$-гладкость и единственность решения начальной задачи (1.1).

Метод $[10,11]$ (см. также [4]) во многом аналогичен подходу $[12,13]$ (развитому применительно к другим ситуациям) и кратко может быть описан следующим образом.

Сделаем замену: от переменной $u(x, t)$ перейдем к переменным $\mathbf{q}=\left(q_{1}, q_{2}, \ldots, q_{n}\right) \in$ $\mathbf{R}^{n}, v=\left(v_{1}, \ldots, v_{n}\right), v_{i} \in L_{2}(\mathbf{R})$. Переменные $q_{i}$ однозначно определяются следующим условием:

$$
\int_{-\infty}^{\infty} \operatorname{ch}^{-2}\left(x-q_{i}\right) v(x, t) d x=\left\langle\frac{\partial U_{i}}{\partial q_{i}}, v_{i}\right\rangle=0,
$$

где $\langle\cdot, \cdot\rangle$ обозначает скалярное произведение в $L_{2}(\mathbf{R})$.

Это условие имеет простой смысл. Мы аппроксимируем решение $u(x, t)$ максимально точно в $L_{2}$-норме с помошью невозмушенных фронтов $(2.2)$, подбирая в каждый момент времени величины $q_{i}$ - координаты (фазы) этих фронтов. Функции же $v_{i}$ описывают искажение $i$-го фронта за счет возмушения $\epsilon f_{i}$.

Итак, весь фронт в целом представляет собой набор кинков и в первом приближении описывается вектор-функцией $\mathbf{U}(x, \mathbf{q})$, зависящей от $x$ и $\mathbf{q}$.

Оказывается, вышеуказанная замена корректно определена при достаточно малых $\epsilon$ в любой малой окрестности $V(C)$, заданной неравенством (2.3). Более того, посредством простых априорных оценок можно доказать, что решение не покидает окрестности $V\left(C_{1}\right)$, где $C_{1}=2 C$.

Это позволяет доказать следующее утверждение [10].

УТВЕРЖДЕНИЕ: 1) существует $c>0$ mакое, что при $t>c|\ln \epsilon|$ и достаточно малых $\epsilon$ в (2.3) можно положить показатель $\kappa=1$, т.е. на больиих временах искажение фронта имеет порядок $\epsilon$;

2) при этом для таких времен выполняются следующие уравнения для $q_{i}(\tau)$ :

$$
\dot{q}_{i}=\tilde{h}_{i}(v, q, \epsilon), \quad \tilde{h}_{i}=h_{i}(q)+\gamma_{i}(v, q, \epsilon),
$$

где $\left|\gamma_{i}\right|<C_{\gamma} \epsilon, \quad t>C|\ln \epsilon|$, а для главных членов правых частей $h_{i}(q)$ верньц формульь

$$
h_{i}=\int_{-\infty}^{\infty} f_{i}(\mathbf{U}(x, \mathbf{q}), x) \operatorname{ch}^{-2}\left(x-q_{i}\right) d x
$$


(В (2.4) и далее точкой обозначается дифференцирование по "медленному времени" $\left.\tau=\frac{3}{4} \epsilon t\right)$.

Заметим, что утверждение 1 иллюстрирует важный эффект взаимодействия нелинейности и диффузии. Мы знаем, что диффузия для линейных проблем обычно приводит к "размыванию” решений. В нелинейном случае она приводит к стабилизации формы фронта волны аналогично тому, как взаимодействие нелинейности и дисперсии для уравнения Кортевега-де Фриза приводит к образованию солитона. Отметим также, что даже немалые начальные искажения фронта $v(x, 0)$ в конце концов исчезают, приводя лишш к сдвигу фазы кинка $[11,14]$.

Таким образом, при малых $\epsilon$ после отбрасывания поправочных членов $\gamma_{i}$ в (2.4) задача сводится к следующей системе уравнений:

$$
\dot{q}_{i}=h_{i}(\mathbf{q})
$$

где $h_{i}$ определяются из выражений (2.5).

Когда система (1.1) описывает распространение волн в однородной среде $\left(f_{i}\right.$ зависит только от $\mathbf{u})$, система (2.6) допускает дальнейшее упрощение, позволяющее понизить ее порядок на единицу. Эта редукция основана на трансляционной инвариантности (1.1), поэтому правые части $h_{k}$ в $(2.6)$ зависят только от относительных разностей координат $q_{i}-q_{j}$.

Введем новые координаты $Q_{i}=q_{i}-q_{n}, i=1, \ldots, n-1$, и вычтем из $i$-го уравнения системы (2.6) (где $i<n-1$ ) уравнение с номером $n$. Тогда уравнения (2.6) принимают вид

$$
\dot{Q}_{i}=H_{i}(\mathbf{Q}), \quad \dot{q}_{n}=h_{n}(\mathbf{Q}),
$$

где $H_{i}=h_{i}-h_{n}, \mathbf{Q}=\left(Q_{1}, Q_{2}, \ldots, Q_{n-1}\right)$ и $i=1, \ldots, n-1$.

Переменные $Q_{i}$ задают относительное расположение кинков. После того как система (2.7) будет решена, можно определить эволюцию координаты центра масс системы кинков $q_{c}=n^{-1}\left(q_{1}+q_{2}+\cdots+q_{n}\right)$.

\section{3. ПЕРИОДИЧЕСКИ ОСЦИЛЛИРУЮШИЕ СВЯЗАННЫЕ СОСТОЯНИЯ КИНКОВ В ОДНОРОДНОЙ СРЕДЕ}

В данном разделе мы приводим простой пример, описывающий некоторые неожиданные эффекты движения волн в однородной среде. Рассмотрим систему (1.1) при $n=3$ и

$$
f_{1}=-u_{3}+\mu, \quad f_{2}=u_{3}+\mu, \quad f_{3}=\left(u_{1}-u_{2}\right)+\mu,
$$

где $\mu$ - не зависимый от $\epsilon$ параметр. Будем искать решение в форме (2.1). Уравнения (2.6) для координат кинков принимают вид

$$
\begin{aligned}
& \dot{q}_{1}=\left\langle U_{3}, U_{1 x}\right\rangle-\mu\left\langle 1, U_{1 x}\right\rangle, \\
& \dot{q}_{2}=-\left\langle U_{3}, U_{2 x}\right\rangle-\mu\left\langle 1, U_{2 x}\right\rangle, \\
& \dot{q}_{3}=\left\langle U_{2}, U_{3 x}\right\rangle-\left\langle U_{1}, U_{3 x}\right\rangle-\mu\left\langle 1, U_{3 x}\right\rangle .
\end{aligned}
$$


Используя очевидные равенства $\left\langle U_{j}, U_{3 x}\right\rangle=-\left\langle U_{3}, U_{j x}\right\rangle,\left\langle 1, U_{j x}\right\rangle=2$, перепишем систему (3.2) в следующей форме:

$$
\begin{aligned}
& \dot{q}_{1}=\left\langle U_{3}, U_{1 x}\right\rangle-2 \mu, \quad \dot{q}_{2}=-\left\langle U_{3}, U_{2 x}\right\rangle-2 \mu, \\
& \dot{q}_{3}=\left\langle U_{3}, U_{1 x}\right\rangle-\left\langle U_{3}, U_{2 x}\right\rangle-2 \mu .
\end{aligned}
$$

Исследуем сначала движение первого и второго кинков относительно третьего. С этой целью, следуя методу, описанному в разделе 2 , введем новые координаты $Q_{1}=q_{1}-q_{3}$, $Q_{2}=q_{2}-q_{3}$. Теперь уравнения (2.7) могут быть записаны в виде

$$
\dot{Q}_{1}=\left\langle U_{3}, U_{2 x}\right\rangle, \quad \dot{Q}_{2}=-\left\langle U_{3}, U_{1 x}\right\rangle
$$

где, как показывают некоторые расчеты, $\left\langle U_{3}, U_{1 x}\right\rangle=F(\alpha),\left\langle U_{3}, U_{2 x}\right\rangle=F(\beta), \quad \alpha=$ th $Q_{1}, \beta=$ th $Q_{2}$, а функция $F$ имеет вид

$$
F(\alpha)=\left(1-\alpha^{2}\right) \int_{-1}^{1} \frac{v d v}{(1-\alpha v)^{2}}=\frac{2}{\alpha}-\frac{2\left(1-\alpha^{2}\right) Q_{1}}{\alpha^{2}} .
$$

Таким образом, динамика относительных координат $Q_{1}, Q_{2}$ описывается следуюшей системой уравнений:

$$
\dot{Q}_{1}=F\left(\operatorname{th} Q_{2}\right), \quad \dot{Q}_{2}=-F\left(\operatorname{th} Q_{1}\right) .
$$

Система (3.6) является консервативной, гамильтоновой, и ее энергия определяется соотношением

$$
E=Q_{1} \operatorname{th}^{-1} Q_{1}+Q_{2} \operatorname{th}^{-1} Q_{2} .
$$

Из (3.7) следует (в силу ограниченности $E$ ), что все решения (3.6) всегда периодические. Выражая $q_{1}, q_{2}, q_{3}$ через $Q_{1}, Q_{2}$ с помощью $(3.5)$ и интегрируя по времени, приходим к формулам

$$
q_{1}=-Q_{2}-2 \mu \tau, \quad q_{2}=-Q_{1}-2 \mu \tau, \quad q_{3}=-Q_{1}-Q_{2}-2 \mu \tau
$$

Последнее уравнение системы (3.8) получено подстановкой (3.4) в третье уравнение (3.3) и последующим интегрированием.

Из (3.8) находим уравнение движения центра масс кинков

$$
\frac{1}{3}\left(q_{1}+q_{2}+q_{3}\right)=-\frac{2}{3}\left(Q_{1}+Q_{2}\right)-\mu \frac{3}{2} \epsilon t .
$$

Следовательно, движение кинков в системе (3.1) осушествляется так: центр масс движется с постоянной средней скоростью, на которую накладываются периодические колебания, при этом кинки также взаимно осциллируют относительно центра масс.

Этот эффект кажется удивительным: ведь исходная нелинейно-диссипативная среда однородна. Однородность среды проявляется в том, что скорость центра масс, усредненная по периоду осцилляций, всегда постоянна и равна $\bar{V}=\frac{3}{2} \epsilon \mu$. При $\mu=0$ возникает связанное состояние из периодически осциллирующих кинков, которые вместе образуют стоячую волну $(\bar{V}=0)$. Возможность данного эффекта связана с тем, что в отличие от многих нелинейных волновых уравнений (и от задач рассеяния многих частиц) 
данная модель описывает "активную" нелинейно-диссипативную среду. Невозможно, следовательно, говорить здесь о законе сохранения полного импульса и даже вообще об импульсе. Отсутствие ограничения на скорость центра масс, связанного с законом сохранения, и приводит к появлению обнаруженного эффекта.

Возникает, однако, вопрос, почему этот эффект не появляется для параболических уравнений второго порядка, которые также описывают аналогичные диссипативные среды. Дело в том, что в этом случае есть другое априорное ограничение на поведение решений. Для уравнения (1.1) (т.е. когда $n=1)$ бо̀льшим начальным данным соответствуют бо̀льшие решения. Данное свойство, хорошо известное как принцип сравнения решений $[5,15]$, является сильным динамическим ограничением, которое запрещает появление нетривиальных волновых решений.

\section{4. ОБРАЗОВАНИЕ ПЕРИОДИЧЕСКИХ ДВИЖУШИХСЯ КИНКОВ В ЛОКАЛЬНО-НЕОДНОРОДНОЙ СРЕДЕ}

Эффекты, полученные в разделе 3 , в случае однородной среды могут возникать только в системах трех и более уравнений. В этом разделе приводится пример возникновения периодических движений фронтов многофазных волн в системе двух уравнений, что становится возможным за счет неоднородности среды. С помощью системы (2.6) мы опишем движение кинков в окрестности локализованной неоднородности. Будет показано, что эти уравнения допускают только периодические решения.

Рассмотрим систему (1.1) с возмушениями $f_{i}$ следуюшего вида:

$$
f_{1}=\rho(x) u_{2}, \quad f_{2}=-\rho(x) u_{1},
$$

где $\rho(x)$ - некоторая функция. Решение этой системы будем искать в форме (2.1). Уравнения (2.6) примут вид

$$
\dot{q}_{1}=-\left\langle\rho U_{2}, U_{1 x}\right\rangle, \quad \dot{q}_{2}=\left\langle\rho U_{1}, U_{2 x}\right\rangle .
$$

Считая, что неоднородность $\rho(x)$ сильно локализована (например, гауссовский пик малой ширины $d$, близкий к $\delta$-функции), приходим к следуюшим выражениям для правых частей системы (4.2):

$$
\left\langle\rho U_{2}, U_{1 x}\right\rangle=\left.\left(U_{2} U_{1 x}\right)\right|_{x=0}+O(d), \quad\left\langle\rho U_{1}, U_{2 x}\right\rangle=\left.\left(U_{1} U_{2 x}\right)\right|_{x=0}+O(d) .
$$

В итоге, пренебрегая членами $O(d)$, видим, что динамика координат кинков будет описываться двумя ОДУ

$$
\dot{q}_{1}=\beta\left(1-\alpha^{2}\right), \quad \dot{q}_{2}=-\alpha\left(1-\beta^{2}\right),
$$

где $\alpha=$ th $q_{1}, \beta=$ th $q_{2}$. Эти уравнения удобно переписать следующим образом:

$$
\dot{\alpha}=\beta\left(1-\alpha^{2}\right)^{2}, \quad \dot{\beta}=-\alpha\left(1-\beta^{2}\right)^{2},
$$

откуда ясно, что они имеют интеграл энергии $E=\left(1-\alpha^{2}\right)^{-1}+\left(1-\beta^{2}\right)^{-1}$. Покажем, что система (4.5) гамильтонова. С этой целью вводим вспомогательную функцию

$$
\phi(\alpha)=\int \frac{d \alpha}{\left(1-\alpha^{2}\right)^{2}}=\frac{\alpha}{2\left(1-\alpha^{2}\right)}+\frac{Q_{1}}{2}
$$


и новые переменные $\phi$ и $\psi$, где $\psi(\beta)=\phi(\beta)$. Обозначая $\alpha=\alpha(\phi), \beta=\alpha(\psi)$, получаем относительно $\phi, \psi$ систему уравнений вида

$$
\dot{\phi}=\beta(\psi), \quad \dot{\psi}=-\alpha(\phi) .
$$

Гамильтониан этой системы определен равенством

$$
H(\psi, \phi)=A(\psi)+A(\phi), \quad A(\psi)=\int \beta(\psi) d \psi .
$$

В силу ограниченности энергии $E$ заключаем, что система (1.1) имеет решения с периодически осциллируюшими фронтами кинков. Поведение решений совершенно аналогично поведению решений, описанному в предыдушем разделе, за исключением того, что нет никакого поступательного движения центра масс: кинки "привязаны" к дефекту.

Другая модель с периодическим движением и нетривиальными эффектами рассеяния может быть получена исходя из результатов предыдушего раздела, если мы рассмотрим нелинейности $\rho(x) f_{i}$, где $f_{i}$ имеют вид (3.1), а функция $\rho \geq 0$ отлична от нуля на некотором интервале длины $L$. Периодические колебания наблюдаются только тогда, когда кинки движутся сквозь неоднородность.

В обоих случаях периодические колебания сохраняются в течение времени $\tau$ порядка $\epsilon^{-1}$, после чего, как показывают численные расчеты и другие соображения (см. ниже раздел 7), колебания затухают. Это связано с малыми поправочными членами $\gamma_{i}$ в $(2.4)$, которые вносят малую диссипацию в (4.5). Результаты следуюшего раздела позволяют построить модель, где периодическое движение не затухает никогда.

\section{5. ХАОТИЧЕСКИЕ ДВИЖЕНИЯ ФРОНТОВ ВОЛН В НЕОДНОРОДНОЙ СРЕДЕ}

Этот раздел будет посвящен модели, в которой могут возникать хаотические (по времени) и незатухаюшие периодические осцилляции фронтов волн. Поскольку вопрос о сушествовании хаоса даже для систем ОДУ является весьма непростым, мы поступим следуюшим образом. Здесь будет построена модель системы, которая сведется (с помошью результатов раздела 2) к системе Лоренца $[16,17]$, для которой существуют хаотические режимы.

Рассмотрим систему (1.1) при $n=3$ и с возмушениями

$$
\begin{aligned}
& f_{1}=\sigma \rho(x)\left(u_{2}-u_{1}\right), \\
& f_{2}=\rho(x)\left(\lambda^{-1} u_{1} u_{3}+r u_{1}-u_{2}\right), \\
& f_{3}=-\rho(x)\left(\lambda^{-1} u_{1} u_{2}+b u_{3}\right),
\end{aligned}
$$

где $\lambda$ - малый параметр, причем $\lambda \gg \epsilon ; \sigma, r, b$ - некоторые параметры. Будем искать решение системы (1.1) в виде (2.1) (при ограничениях (2.2), (2.3) на начальные данные). В результате из (2.6) получаем уравнения, описывающие эволюцию координат кинков,

$$
\begin{aligned}
& \dot{q}_{1}=\sigma\left(\left\langle\rho U_{1}, U_{1 x}\right\rangle-\left\langle\rho U_{2}, U_{1 x}\right\rangle\right), \\
& \dot{q}_{2}=-\lambda^{-1}\left\langle\rho U_{1} U_{3}, U_{2 x}\right\rangle-r\left\langle\rho U_{1}, U_{2 x}\right\rangle+\left\langle\rho U_{2}, U_{2 x}\right\rangle, \\
& \dot{q}_{3}=\lambda^{-1}\left\langle\rho U_{1} U_{2}, U_{3 x}\right\rangle+b\left\langle\rho U_{3}, U_{3 x}\right\rangle .
\end{aligned}
$$


При тех же предположениях о $\rho(x)$, что и в разделе 4 , приходим к следующей системе уравнений, описывающей динамику фронтов кинков:

$\dot{q}_{1}=\sigma(\beta-\alpha)\left(1-\alpha^{2}\right), \quad \dot{q}_{2}=\left(r \alpha-\beta-\lambda^{-1} \alpha \gamma\right)\left(1-\beta^{2}\right), \quad \dot{q}_{3}=\left(\lambda^{-1} \alpha \beta-b \gamma\right)\left(1-\gamma^{2}\right)$,

где $\alpha=\operatorname{th} q_{1}, \beta=\operatorname{th} q_{2}, \gamma=\operatorname{th} q_{3}$. Из-за сложности системы (5.3) мы изучим поведение ее решений только в малой окрестности нуля $\mathbf{q}=0$ размером $O(\lambda)$. Мы собираемся показать, что данные решения не покидают эту окрестность и их поведение внутри нее с точностью до малых поправок определяется системой Лоренца.

Введем новые переменные $Q_{i}=\lambda^{-1} q_{i}$. Тогда с точностью до $O\left(\lambda^{2}\right)$ уравнения (5.3) принимают вид

$$
\dot{Q}_{1}=\sigma\left(Q_{2}-Q_{1}\right), \quad \dot{Q}_{2}=r Q_{1}-Q_{2}-Q_{1} Q_{3}, \quad \dot{Q}_{3}=Q_{1} Q_{2}-b Q_{3}
$$

Уравнения (5.4) совпадают с уравнениями системы Лоренца, для которой при определенном выборе параметров $\sigma, r, b$ существуют хаотические решения.

Чтобы оправдать переход от (5.3) к (5.4), необходимы некоторые дополнительные рассуждения. Во-первых, мы должны найти такую окрестность нуля $D \subset \mathbf{R}^{3}$, при которой если в начальный момент $\mathbf{Q}$ лежит в $D$, то и во все последующие моменты это так. Тогда $Q_{i}$ всегда будут оставаться ограниченными, и, следовательно, всегда можно (для таких начальных данных) перейти от (5.3) к (5.4). Такая инвариантная область $D$ существует благодаря диссипативности системы Лоренца и имеет вид

$$
D=\left\{\left(Q_{1}, Q_{2}, Q_{3}\right) \mid Q_{1}^{2}+Q_{2}^{2}+\left(Q_{3}-\sigma-r\right)^{2} \leq c^{2}\right\}
$$

где постоянная $с$ должна быть достаточно велика [18]

Во-вторых, если мы желаем иметь строгое доказательство сушествования хаоса, необходимо также показать, что малые отброшенные члены $O\left(\lambda^{2}\right)$ и $\gamma_{i}$ не вносят качественных изменений в поведение решений. Это весьма тонкая и трудная задача. Однако в последнее время появился ряд работ, где разработан специальньй метод, позволяющий доказывать существование хаотических режимов для УЧП [19-23]. Такие доказательства содержат ряд дополнительных математических деталей, которые делают их довольно длинными.

В данной работе мы опускаем детальные рассуждения. Решаюшее обстоятельство, позволяющее добиться успеха, заключается в следующем. Согласно [16] система Лоренца обладает некоторыми свойствами структурной устойчивости. Именно, с помощью сечения Пуанкаре с ней стандартным образом можно связать некоторое отображение. Это отображение меняется при малом возмущении, но сохраняет хаотические свойства, поскольку оно может быть включено в структурно-устойчивый двухпараметрический класс хаотических отображений [16].

Отметим, что, поскольку в модели Лоренца также возможны грубые (структурноустойчивые) периодические циклы, одновременно мы получаем пример системы, где в отличие от примеров, приведенных в разделах 3,4 , периодическое движение сохраняется неограниченно долго. 


\section{6. РАССЕЯНИЕ КИНКОВ ПРИ ПЕРЕХОДЕ ГРАНИЦЫ СРЕД С РАЗЛИЧНЫМИ СВОЙСТВАМИ}

Рассмотрим модель рассеяния волн при переходе гранишы двух различных сред. Пусть в системе (1.1) функции $f_{i}$ определены выражениями

$$
f_{1}=-2-\eta(x) u_{2}, \quad f_{2}=-2,
$$

где $\eta(x)$ - функция Хевисайда, равная 1 при $x>0$. Уравнения (2.6) при возмушениях (6.1) имеют вид

$$
\dot{q}_{1}=2\left\langle 1, U_{1 x}\right\rangle+\left\langle\eta U_{2}, U_{1 x}\right\rangle, \quad \dot{q}_{2}=2\left\langle 1, U_{2 x}\right\rangle
$$

После некоторых несложных вычислений система (6.2) преобразуется к следующей:

$$
\dot{q}_{1}=4+F(\alpha, \delta), \quad \dot{q}_{2}=4,
$$

где $\alpha=\operatorname{th}\left(q_{1}\right), \delta=\operatorname{th}\left(q_{1}-q_{2}\right)$;

$$
F(\alpha, \delta)=\frac{1+\alpha}{\delta}+\frac{1-\delta^{2}}{\delta^{2}} \ln \left(\frac{1-\alpha \delta}{1+\delta}\right), \quad \delta \neq 0, \quad F(\alpha, 0)=\frac{1-\alpha^{2}}{2} .
$$

Поскольку $|F(\alpha, \delta)|<2$, то ясно, что обе волны движутся в положительном направлении, причем $q_{2}=4 \tau+q_{2}(0), 2<\dot{q}_{1}<6$.

Рассмотрим задачу Коши для уравнений (6.3) с начальными данными $q_{1}(0)=$ $q_{2}(0) \ll-1$. Тогда, как видно из выражения (6.4), динамика кинков такова: сначала волны движутся вместе с почти одинаковой скоростью, поскольку $F$ мало при больших отрицательных $q_{1}$. Рассмотрим динамику относительного расстояния $Q=q_{1}-q_{2}$ между кинками при больших положительных $q_{1}$ (кинки всегда проникают в эту область согласно нашей оценке их скоростей). В этом случае из (6.4) следует

$$
\dot{Q}=\frac{2}{\operatorname{th} Q}\left(1-\frac{Q}{\operatorname{ch} Q \operatorname{sh} Q}\right) .
$$

Из этого уравнения вытекает, что $Q(t) \rightarrow \infty$ при $t \rightarrow \infty$, и, следовательно, кинки рассеиваются. Расстояние между ними увеличивается неограниченно, и при больших временах мы получаем два независимых кинка, движушихся с различными скоростями. Таким образом, данная модель описывает эффект распада связанного состояния кинков при изменении свойств среды.

\section{7. НЕКОТОРЫЕ РЕЗУЛЬТАТЫ ЧИСЛЕННЫХ ЭКСПЕРИМЕНТОВ}

Прямые численные расчеты исходной системы (1.1) полностью подтверждают результаты разделов 3,4 и 6 (система из раздела 5 нами не рассматривалась из-за отсутствия достаточно мошного компьютера) и, кроме того, обнаруживают следующую любопытную закономерность. При численном исследовании системы в разделе 4, когда $\epsilon$ не мало, пространственная структура фронта нарушается и временные осцилляции становятся затухаюшими. Кинки сближаются к устойчивому положению равновесия 
$q_{1}=q_{2}=0$. При этом время затухания тем меньше, чем больше параметр $\epsilon$. Это может быть объяснено следующими соображениями.

Фазовый портрет систем (2.6), рассмотренных в разделах 3,4 , состоит из набора замкнутых кривых, топологически эквивалентных окружностям, окружаюшим точку равновесия $q_{1}=q_{2}=0$. Как показывают компьютерные вычисления, эта точка равновесия, соответствуюшая стационарному решению $u_{i}=$ th $x, i=1,2$, всегда устойчива независимо от выбора параметра $\epsilon$. Данньй факт может быть объяснен и аналитически с помошью исследования квадратичной формы линеаризованного оператора и известных результатов $[14,15]$.

Таким образом, отброшенные поправочные члены $\gamma_{i}$ в (2.6) являются, как и следовало ожидать, диссипативными. Это и приводит к медленному затуханию (за время порядка $O\left(\epsilon^{-2}\right)$ периодического режима в системах, приведенных в разделах 3,4 .

\section{8. ЗАКЛЮЧЕНИЕ}

Данная работа показывает, что даже в самых простых моделях с полиномиальными нелинейностями не выше кубической возможно сложное взаимодействие нелинейных волн. Однако в данной одномерной модели размерность аттрактора системы ОДУ (2.6), определяющей поведение координат кинков, ограничена сверху числом компонент $n$. По-видимому, в двумерных двухкомпонентных системах с такими же простыми нелинейностями взаимодействие уединенных волн может породить неограниченно сложное поведение.

\section{Список литературы}

[1] А.Н. Колмогоров, Г. И. Петровский, Н. С. Пискунов. Бюлл. МГУ. Сер. А. 1937. Т. 1. № 6. C. 1.

[2] В. П. Маслов, К.А. Волосов, В. Г. Данилов. Математические проблемы тепломассопереноса. М.: Наука, 1987.

[3] Y. Kuramoto. Chemical oscillations, waves and turbulence. Berlin: Springer, 1984.

[4] И. А. Молотков, С. А. Вакуленко. Сосредоточенные нелинейные волны.. Л.: Изд-во ЛГУ, 1988.

[5] А.А. Самарский, В.А. Галактионов, С.П. Курдюмов, А.П. Михайлов. Режимы с обострением в задачах для квазилинейных параболических уравнений. М.: Наука, 1987.

[6] Джс. Марри. Нелинейные дифференциальные уравнения в биологии. Лекции о моделях. М.: Мир, 1983.

[7] А.И. Вольперт. Волновые решения параболических уравнений. Препринт института химической физики АН СССР. Черноголовка, 1983.

[8] B. A. Вольперт. Некоторые вопросы устойчивости бегущих волн. Препринт института химической физики АН СССР. Черноголовка, 1980.

[9] В. А. Вольперт, А.И. Вольперт. Некоторые приложения функционального анализа к задачам математической физики. Т. 145. Новосибирск: Сиб. отд. ин-та математики АН СССР, 1990. C. 49.

[10] С. А. Вакуленко. ЖВМ и МФ. 1991. Т. 31. № 5. С. 735.

[11] S. A. Vakulenko. Nonlinear Analysis TMA. 1992. V. 19. P. 1033.

[12] G. A. Fusco. Geometric approach to the dynamics of $u_{t}=\epsilon^{2} u+f(u)$. In: Problems involving change of type. Lect. Notes in Phys. V. 359. Berlin-Heidelberg: Springer, 1990. P. 53-73.

[13] J. Carr, R. Pego. Very slow phase separation in one dimension. In: PDEs and continuum models of phase transition. Lect. Notes in Phys. V. 344. Eds M. Rasele et al. Berlin-Heidelberg: Springer, 1989. P. 216. 
[14] P. C. Fife, J. B. Macleod. Arch. Rat. Mech. Anal. 1977. V. 65. P. 335.

[15] Д. Хенри. Геометрическая теория полулинейных параболических уравнений. М.: Мир, 1985.

$[16]$ Д. В. Аносов, С.Х. Арансон и др. Итоги науки и техники. Сер. Современные проблемы математики. Т. 66. М.: ВИНИТИ, 1991.

[17] В.И. Арнольд, В.С. Афраймович, Ю. С. Ильяшенко, Л. П. Шильников. Итоги науки и техники. Сер. Современные проблемы математики. Т. 5. М.: ВИНИТИ, 1985.

[18] Э. Хайрер, С. Нёрсетm, Г. Ваннер. Решение обыкновенных дифференциальных уравнений. М.: Мир, 1990.

[19] P. Polacik. Ann. Scuole Norm Pisa. 1991. V. 17. P. 83.

[20] B. Fiedler, P. Polacik. Proc. Roy. Soc. of Edinburgh. 1990. V. 115. P. 167.

[21] S. A. Vakulenko. C. R. Acad. Sci. Paris. 1993. V. 316. Ser. I. P. 1015.

[22] S. A. Vakulenko. J. Phys A: Math. Gen. 1994. V. 27. P. 2335.

[23] S. A. Vakulenko. Ann. Inst. Henri Poincare, Phys. Theor. 1997 (to appear).

Поступила в редакцию 24.III.1997 г.

\section{S. A. Vakulenko, P. V. Gordon \\ PROPAGATION AND SCATTERING OF KINKS IN INHOMOGENEOUS NONLINEAR MEDIA}

The model of the kink scattering in inhomogeneous nonlinear media is considered. The existance of time periodically and chaotically oscillating kink bound states is shown. 